\title{
Criminologie
}

\section{Les policiers, la Loi sur les jeunes contrevenants et le système de justice pour mineurs}

\section{Marc LeBlanc}

Volume 17, numéro 1, 1984

La police après 1984

URI : https://id.erudit.org/iderudit/017192ar

DOI : https://doi.org/10.7202/017192ar

Aller au sommaire du numéro

Éditeur(s)

Les Presses de l'Université de Montréal

ISSN

0316-0041 (imprimé)

1492-1367 (numérique)

Découvrir la revue

Citer cet article

LeBlanc, M. (1984). Les policiers, la Loi sur les jeunes contrevenants et le système de justice pour mineurs. Criminologie, 17(1), 91-115.

https://doi.org/10.7202/017192ar d'utilisation que vous pouvez consulter en ligne.

https://apropos.erudit.org/fr/usagers/politique-dutilisation/ 


\section{LES POLICIERS, LA LOI SUR LES JEUNES CONTREVENANTS ET LE SYSTÈME DE JUSTICE POUR MINEURS \\ Marc LeBlanc*}

1908-1983: soixante-quinze ans pendant lesquels la Loi fédérale sur les jeunes délinquants a été en application. 1957-1983: vingtsix ans pendant lesquels cette loi a été soumise à diverses tentatives de révision.

Ces longues périodes sont un indice de la haute qualité d'une législation qu'il n'y avait pas lieu d'adapter aux transformations de la société et au développement des connaissances. Mais elles peuvent aussi signifier l'incapacité des gouvernements, et des intervenants de toute origine, à s'entendre sur le contenu d'une nouvelle loi à voter. Départager les raisons de l'inaction du législateur n'est pas le propos de cet article. Plutôt, nous nous intéresserons aux réactions et opinions des policiers sur la nouvelle Loi fédérale sur les jeunes contrevenants. Voyons comment cette nouvelle législation, votée en mai 1982, est reçue par des policiers expérimentés dans l'application de l'ancienne Loi sur les jeunes délinquants.

\section{UNE NOUVELLE JUSTICE POUR MINEURS}

La Loi sur les jeunes contrevenants propose des transformations majeures pour le système de justice pour mineurs parce qu'elle s'inspire de principes totalement différents de ceux de l'ancienne Loi fédérale sur les jeunes délinquants. Voici comment le ministère du Solliciteur général du Canada présente ces modifications (1982):

Elle (la loi) met en cuvre une conception fondée sur les droits et responsabilités des jeunes qui ont des démêlés avec la justice. D'une part, elle met l'accent sur l'idée que les adolescents doivent être tenus responsables de leur conduite illicite et que la société a le droit de se protéger contre une telle conduite. D'autre part, elle reconnaît que les adolescents ont des besoins spéciaux et qu'ils ne sauraient pas, dans tous les cas, être assimilés aux adultes quant à leur degré de développement et de maturité. Compte tenu du droit de la société, de se protéger et des besoins spéciaux des adolescents, ils ont non seulement besoin

Cette enquête a été réalisée grâce à un contrat du ministère des Aprovisionnements et Services, sous la direction du ministère du Solliciteur général du Canada dans le cadre de l'étude nationale sur le fonctionnement des tribunaux pour mineurs.

* Professeur titulaire, École de criminologie, chercheur au Centre international de criminologie comparée, Université de Montréal. 
de surveillance, de discipline et d'encadrement, mais également de conseils et d'assistance ainsi que de garanties spéciales pour leurs droits fondamentaux: ..., p. 2.

En somme, cette nouvelle législation sonne le glas de la doctrine du «parens patriae» dans nos tribunaux pour mineurs, l'État n'est plus le bon père de famille qui propose son aide à l'enfant mal dirigé. On instaure plutôt un modèle de justice qui accorde des droits fondamentaux aux adolescents et qui propose des procédures et des décisions qui diffèrent de celles qui existent pour les adultes. On ne veut plus uniquement traiter les jeunes délinquants, on propose aussi de protéger la communauté contre leurs agissements nuisibles.

\section{DES ACTEURS À CONSULTER}

Cette nouvelle philosophie modifiera les pratiques des policiers, avocats, juges, intervenants sociaux, experts, ... Nous avons été amenés à demander à des policiers ce qu'ils pensent de la nouvelle philosophie proposée, est-ce qu'ils approuvent les objectifs proposés pour la justice pour mineurs. Et nous avons été en mesure d'aller plus loin et de vérifier s'ils appuient les transformations que subira la justice pour mineurs dans sa quotidienneté, ceci à travers leur approbation des procédures mises de l'avant.

Cette démarche poursuit à la fois un but pédagogique et d'évaluation. En effet, l'identification des zones d'ambivalence pourra nous indiquer des cibles de formation et d'information. Tandis que les zones de résistance indiqueront autant de principes et de procédures qui risquent d'être court-circuités par les policiers dans leur pratique. Par ailleurs, l'opinion des policiers, avant que l'information sur la loi ne soit diffusée et que l'implantation ne soit réalisée, constitue une donnée de base nous permettant, dans les années à venir, de confronter l'évolution des pratiques et ainsi d'évaluer l'écart entre les principes et les actions courantes des intervenants policiers.

\section{DES POLICIERS SE PRONONCENT SUR LA LOI SUR LES JEUNES CONTREVENANTS}

Ả l'été 1982, immédiatement après que la Loi sur les jeunes contrevenants eut été votée, nous avons administré un questionnaire $^{2}$ de trente pages à des policiers de la Communauté

2. Le questionnaire a été construit, dans le cadre de l'étude nationale sur le fonctionnement des tribunaux pour mineurs, sous la direction d'Aaron Caplan et avec la collaboration principale de Ray Corrado et Rick Linden. 
urbaine de Montréal $^{3}$. Cette loi n'était pas inconnue de la plupart d'entre eux, puisque $66,2 \%$ avaient déjà lu des documents la concernant, mais seulement $37,9 \%$ avaient participé à des séances internes d'information sur cette loi.

La direction de la police de la C.U.M. a constitué un échantillon aléatoire de 250 policiers dont environ une centaine de la section jeunesse et relations communautaires et le reste tiré parmi les détectives et patrouilleurs auxquels se sont ajoutés plusieurs officiers supérieurs. La poste interne du service a été utilisée pour les envois et retours, ceci sous enveloppes scellées. Parmi les 250 questionnaires transmis, 141 sont revenus rapidement et 109 policiers ont fait l'objet d'un rappel. 195 questionnaires ont été complétés, 78,3\% des policiers contactés y ont donc répondu.

$98 \%$ des policiers qui ont répondu étaient de sexe masculin, ils avaient entre 31 et 59 ans pour une moyenne de 41,18 ans et $90,3 \%$ étaient mariés; ils étaient pour la plupart père, dont $11 \%$ d'un enfant, $55 \%$ de deux enfants et $24 \%$ de trois enfants. Il s'agit d'un groupe dont la scolarisation est élevée car $52 \%$ ont entrepris des études collégiales, pour $24 \%$ d'entre eux, elles ne sont pas terminées et pour $28 \%$ un diplôme collégial a été obtenu. $6 \%$ des policiers interrogés possèdent un diplôme universitaire. Ils ont aussi une longue expérience du travail policier, en moyenne 18 ans.

Parmi les répondants, $50,3 \%$ sont des agents, $30,6 \%$ des sergents $(24,4 \%)$ et sergents détectives $(6,2 \%) 15 \%$ des lieutenants $(11,4 \%)$ et lieutenants détectives $(3,6 \%)$ et $4,1 \%$ sont des officiers de grades supérieurs. De plus $61 \%$ des représentants affirment faire partie de la section d'aide à la jeunesse et ce, depuis en moyenne douze ans. Ces policiers se sont, pour la plus grande part, déjà présentés devant le tribunal de la jeunesse $(93,4 \%$ ) et la plupart d'entre eux $(80 \%)$, au cours des quatre dernières années.

$86 \%$ des répondants déclarent aimer leur travail et ce, parce qu'ils aident les jeunes et qu'ils sont en contact avec la jeunesse. Leurs sources d'insatisfaction sont les défauts du système de justice, dont la Loi provinciale sur la protection de la jeunesse, et les critiques que les autres professionnels et le public énoncent à leur égard.

En somme, les répondants possèdent une expérience professionnelle importante et personnellement significative avec les jeunes.

3. Nous remercions les autorités de la police de la C.U.M. pour leur excellente collaboration à la cueillette des données. 
Ils représentent un groupe scolarisé issu de toutes les couches du service de police et ils sont satisfaits de leur travail. Notons finalement que l'utilité pédagogique et évaluative de ce sondage que nous mettions de l'avant plus tôt, est évidente lorsque les policiers déclarent que la nouvelle loi ne changera pas leur travail $(30 \%)$, qu'elle le modifiera en mieux $(27 \%)$, qu'elle l'altèrera pour le rendre plus difficile $(10 \%)$ et que $35 \%$ se disent indécis quant à l'impact de la nouvelle loi fédérale sur leur travail.

\section{ILS APPROUVENT DE FAÇON MITIGÉE LA PHILOSOPHIE DE LA LOI}

La déclaration de principe de la Loi sur les jeunes contrevenants est ainsi rédigée que chaque principe s'affirme comme la recherche d'un équilibre entre deux positions que l'on tend généralement à opposer, par exemple la protection de la société et les besoins des jeunes, chaque principe est énoncé de manière à introduire des circonstances qui en atténuent la portée, par exemple les jeunes contrevenants doivent assumer la responsabilité de leurs actes mais ils ne sauraient être assimilés aux adultes quant à leur degré de responsabilité et aux conséquences de leurs actes. Cette rédaction des principes de la Loi sur les jeunes contrevenants nous amène à penser que pour approuver la philosophie de la loi, les policiers devraient donner une importance équivalente aux deux éléments d'un même principe.

L'analyse du Tableau $1^{4}$ nous révèle que le degré d'approbation de la philosophie de la Loi sur les jeunes contrevenants est mitigée; en effet $69 \%$ des policiers n'approuvent pas l'octroi des garanties spéciales aux adolescents en ce qui concerne leurs droits et libertés; pas beaucoup plus de la moitié reconnaissent le droit des adolescents à l'intervention minimale $(\mathbf{5 8 \%}$ ) ou non-assimilation aux adultes quant aux conséquences à leurs actes $(57 \%)$; deux tiers à trois quarts d'entre eux sont d'accord de ne pas considérer les adolescents comme des adultes quant à leurs responsabilités $(65 \%)$, de leur accorder le droit de participer au processus de décision (64\%) et de les soustraire le moins possible à l'autorité parentale $(72 \%)$. Par ailleurs, ils approuvent l'affirmation à l'effet que les adolescents doivent être tenus responsables $(\mathbf{7 4 \%})$ et ils préfèrent que la protection de la société prédomine sur les besoins des adolescents $(83 \%)$ ce qui peut être interprété comme une option principale de la nouvelle loi car la protection de la société est souvent une circonstance qui qualifie les principes plus généreux à l'égard des adolescents.

4. Notons que tous les tableaux auxquels nous référerons dans le texte sont placés, selon leur ordre de numération, en annexe. 
L'appui à la philosophie de la nouvelle loi est d'autant plus mitigé qu'environ un tiers des policiers, à toutes les questions, se situent dans la catégorie qui marque un degré d'ambivalence évident, plutôt d'accord et plutôt en désaccord. De plus l'équilibre entre les composantes des principes penche systématiquement en faveur de la protection de la société plutôt qu'à l'égard de la protection des jeunes. Par exemple $\mathbf{9 4 \%}$ des policiers sont d'accord pour déclarer que des adolescents doivent être tenus responsables de leurs actes tandis que respectivement $65 \%$ et $57 \%$ soutiennent qu'ils ne sauraient être assimilés aux adultes quant à leur degré de responsabilité et aux conséquences de leurs actes. Cet écart indique donc que l'équilibre que les policiers proposent entre les divers principes directeurs de la nouvelle législation n'est peut être pas celui que le législateur espérait obtenir, «concilier, selon les termes du ministre Kaplan (1982), les intérêts de la société, le principe de responsabilité des adolescents ainsi que la nature particulière des besoins et des droits des jeunes».

Enfin, nous pouvons soutenir que les policiers approuvent de façon mitigée la philosophie de la nouvelle loi parce que si nous donnons à chaque policier un score d'approbation en faisant la sommation de ses réponses à toutes les questions à ce sujet, nous notons que l'approbation totale serait un score de 9 , une approbation très marquée serait un score de 18 et une approbation faible signifierait un score de 27. Leur approbation de la philosophie est mitigée car le score moyen des policiers est de 22 . En somme, ils approuvent les principes de la nouvelle législation mais de façon timide. Ce qu'ils soutiennent de toute évidence c'est un équilibre où la protection de la société aurait une domination incontestable tout en laissant une place subsidiaire aux besoins des jeunes et aux circonstances atténuantes de leur développement inachevé.

\section{TOUT EN RESTANT PRUDENTS FACE À CERTAINES OPTIONS DE LA NOUVELLE LOI}

Ainsi, la Loi sur les jeunes contrevenants fixe à 12 ans l'âge minimal de la responsabilité criminelle et à 18 ans l'âge maximal. Pour les policiers, l'âge minimal est jugé adéquat par $59 \%$ d'entre eux, d'autres préfèrent 14 ans $(28 \%)$ et le reste $(13 \%)$ d'autres âges, principalement inférieurs à 12 ans. L'âge maximal choisi ne fait pas consensus car $52 \%$ d'entre eux préfèraient 16 ans contre $34 \% 18$ ans et $14 \%$ pour des âges inférieurs à 14 ans. Il conviendra, lors de l'implantation, de mieux justifier ces choix des législateurs et d'expliciter adéquatement les raisons qui les motivent, ce que fait la Commission Charbonneau (1982). Par ailleurs, la plupart des policiers (72\%) 
sont d'accord pour extraire de la Loi fédérale les infractions de situation (promiscuité, fréquentation scolaire, ...) et laisser les provinces légiférer à cet égard.

Comme pour l'âge, dans le domaine de la durée des sentences, les policiers se démarquent de ce que propose la nouvelle législation. Ainsi pour un crime passible d'une sentence à perpétuité, les policiers préfèrent une sentence d'une durée de cinq ans $(49 \%)$ à la durée prévue de 3 ans, celle-ci ne recueille l'appui que de $15 \%$ des policiers. Pour les autres infractions, la nouvelle loi prévoit une durée de la sentence de deux années, ce qu'approuvent les policiers à $51 \%$, toutefois $22 \%$ opteraient pour cinq ans. En somme, les policiers semblent d'accord sur le changement par lequel les durées indéterminées des sentences seront remplacées par des durées spécifiques maximales; toutefois ils préfèreraient, pour les crimes graves, que ces durées soient plus longues. Quant aux montants des amendes, le montant de $\$ 1000,00$ leur apparaît généralement approprié $(51 \%)$.

En somme, un bon nombre de policiers se sentent mal à l'aise de devoir traiter différemment des adultes, les contrevenants de 16 à 18 ans qu'ils préfèreraient voir assimilés à ceux-là. Ils favoriseraient aussi des durées de sentences plus longues que celles prévues par la Loi, surtout en ce qui concerne les crimes les plus graves. Ces options sont tout à fait congruentes avec leur préférence pour la protection de la société que nous avons mis en exergue précédemment.

\section{ET EN APPUYANT AVEC PLUS DE VIGUEUR LES PROCÉDURES QUE PROPOSE LA LÉGISLATION}

La comparaison des pourcentages d'approbation, aux tableaux 1 et 2 (en annexe), montre, que de façon générale, les policiers donnent un appui plus évident aux mesures substantives et procédurales prévues dans la Loi sur les jeunes contrevenants qu'ils ne le font pour sa philosophie et ses cadres (âge, durée des sentences). Cette observation vient montrer que l'écart entre l'approbation inconditionnelle et l'acceptation mitigée de la nouvelle législation est une question d'accent plutôt que le résultat d'une différence fondamentale d'approche au problème de la délinquance des mineurs. Voyons leur position par rapport aux principales mesures substantives et procédurales, ceci toujours en distinguant celles qui se rapportent directement au travail des policiers et celles qui s'appliquent à d'autres composantes du système de justice.

Nous avons noté précédemment que seulement un peu plus de la moitié des policiers interrogés acceptent que l'on reconnaisse aux adolescents le droit à l'intervention minimale. Malgré cette position 
de principe, ils acceptent mieux les mesures de rechange, généralement $75 \%$ d'entre eux, dans le cadre de procédures spécifiques:

- si l'adolescent a librement manifesté sa ferme volonté de collaborer à leur mise en cuvre et s'il ne manifeste pas le désir de se voir déféré au Tribunal de la jeunesse;

- s'il y a des preuves suffisantes justifiant des poursuites; cette position vient confirmer le rôle du procureur de la couronne dans cette démarche;

- en autant que le recours à ces mesures n'empêche pas la mise en cuvre de poursuites relatives à l'infraction.

Cependant les policiers, au moins $42 \%$ d'entre eux, proposent que de telles mesures peuvent être appliquées même si l'adolescent nie toute participation à l'infraction; cette position apparaît quelque peu surprenante. Parallèlement, 52\% d'entre eux ne sont pas d'accord avec la position de la Loi qui encourage le juge à rejeter les accusations lorsque l'adolescent a entièrement accompli les mesures de rechange et, corollairement, $83 \%$ ne veulent pas que l'accusation soit rejetée lorsque l'adolescent les a partiellement accomplies. Ainsi, ils réaffirment leur position de principe favorisant la conséquence à l'acte et surtout la conséquence entière sans adoucissement qui risquerait de rendre les mesures de rechange moins punitives et qui créerait une image de laxisme.

En ce qui concerne certaines procédures qui se rapportent plus directement au travail des policiers, notons que la quasi-totalité des policiers, $(\mathbf{9 2 \%}$ ) pensent qu'il ne convient pas de détenir un mineur dans le même local que des délinquants adultes et plus des trois quarts $(\mathbf{7 8 \%})$ préfèrent que la mise en liberté sous caution se fasse selon les règles et critères du Code Criminel. Ils sont convaincus $(96 \%)$ qu'un avis aux parents doit être donné le plus rapidement possible lors de l'arrestation et/ou de la détention et ils pensent $(86 \%)$ qu'au tribunal, le juge doit ajourner l'affaire si un avis n'a pas été donné. Ils sont toutefois plus réticents à ce que les procédures soient invalidées si l'avis n'a pas été donné ( $46 \%$ sont d'accord pour rendre caduque la procédure).

Si $85 \%$ des policiers sont d'accord pour que l'on assure les services d'un avocat à l'adolescent devant le tribunal, ils sont plus réticents à accepter la représentation à toutes les phases des poursuites; $73 \%$ seraient prêts à le faire. En effet, les trois quarts seraient prêts à informer l'adolescent de ce droit et à lui laisser la chance de l'exercer. 
Cette proportion est moindre que celle représentant l'approbation de la représentation légale à la phase du tribunal, toutefois l'écart n'est pas très grand.

Les policiers $(\mathbf{8 4 \%})$ sont d'accord pour prendre les empreintes digitales ou la photographie d'un mineur lorsqu'un acte criminel est imputé à un adolescent. Toutefois, en ce qui concerne les infractions punissables sur déclaration sommaire de culpabilité, seulement $61 \%$ seraient prêts à procéder à ces opérations d'identité policière.

Concernant les décisions, la quasi-totalité des policiers ne veulent pas que les adolescents soient soumis à des peines plus graves que celles prévues pour les adultes; ils sont entièrement d'accord à remplacer les amendes par des travaux communautaires; et ils préfèrent nettement que ce soient les juges qui déterminent le niveau approprié de placement sous garde, à savoir le choix entre un milieu sécuritaire et non sécuritaire. Par ailleurs, une légère majorité des policiers, $55 \%$, s'opposent à ce que ce soient les autorités provinciales, les services sociaux, qui choisissent l'endroit approprié de placement, le centre d'accueil. Beaucoup pensent que le tribunal devrait avoir une autorité totale sur cette décision. Quant à l'examen des décisions, les révisions, ils adoptent une position tout à fait congruente à celle retenue pour les décisions elles-mêmes: $91 \%$ préfèrent que le tribunal garde juridiction, mais ils acceptent que le procureur de la couronne $(83 \%)$, les parents des jeunes $(78 \%)$, l'adolescent lui-même $(68 \%)$ et les services sociaux $(61 \%)$ puissent demander une révision au tribunal. De fait, ils sont divisés ( $55 \%$ pour, $45 \%$ contre) quant à l'opportunité de mettre sur pied une commission provinciale qui réexaminerait les décisions comportant un placement sous garde. Quant au caractère public des audiences, $61 \%$ des policiers optent pour des audiences privées.

Le dernier point, sur lequel nous avons interrogé les policiers, concerne les dossiers. La Loi sur les jeunes contrevenants s'étend longuement sur cette question. Les policiers $(65 \%)$ ne sont pas d'accord pour que les dossiers de l'adolescent soient à sa disposition et corollairement, tous préfèrent que le juge régularise l'accès au dossier tout en spécifiant ce qui ne devrait pas être mis à la disposition du mineur. Quant à la destruction des dossiers, ils sont encore plus réticents à accepter les propositions de la nouvelle Loi sur les jeunes contrevenants. En effet, $64 \%$ pensent que les dossiers d'infractions punissables par déclaration sommaire de culpabilité ne devraient pas être détruits même s'il n'y a pas eu de récidive deux ans après l'exécution complète de la mesure. En ce qui concerne les dossiers où appa- 
raissent des actes criminels, sans récidive depuis cinq ans après l'exécution des mesures, $46 \%$ pensent qu'il ne faut pas les détruire.

Au terme de cette section sur les mesures substantives et procédurales, il est bien évident que les policiers s'y acclimateront plus facilement qu'aux principes mêmes de la nouvelle loi. S'ils demeurent bien disposés à les appliquer, il est aussi manifeste, qu'en ce qui concerne leur travail, ils préfèrent le moins de contraintes possible, de contrôles, qui risquent de leur amener des critiques. Critiques qui sont, nous l'avons vu, une des sources majeures d'insatisfaction dans leur travail.

\section{DES POLICIERS JAUGENT LE SYSTĖME DE JUSTICE POUR MINEURS}

Le questionnaire qui a été administré aux policiers comportant plusieurs questions sur l'efficacité du système de justice pour mineurs, mais avant de présenter leur appréciation de celle-ci, il convient de regarder leur perception des phénomènes de la délinquance et la description qu'il font de leur action.

\section{1. À partir d'une perception réaliste du phénomène de la délinquance}

La moitié des policiers placent au premier rang le vol à main armée comme le crime le plus grave que commettent les adolescents dans leur communauté; au second rang, avec $21,5 \%$ des choix, vient le vol avec effraction (voir tableau 3 , en annexe). Comme deuxième choix, on retrouve, le vol avec effraction ( $34 \%$ des choix) suivi sur un pied d'égalité (18 et 19\%) de la violence en général, les délits contre les biens et le vol à main armée. Pour les choix suivants, troisième et quatrième, on voit une prédominance marquée des délits contre les biens, respectivement $70 \%$ et $66 \%$. Il convient de noter que les policiers mentionnent à tous les choix, avec un effectif d'environ 8 à $10 \%$, la prostitution et la drogue comme des problèmes sociaux.

Si les policiers mettent un accent sur la criminalité traditionnelle, ils ont une vue plutôt réaliste du phénomène (tableau 4 , en annexe) car $51 \%$ pensent que de 5 à $30 \%$ des jeunes constituent une menace réelle pour la société, ce qui coïncide avec les travaux de Fréchette et LeBlanc (1979-1980) qui étudient le développement de la délinquance et qui, par leurs travaux, situent dans ce champ la délinquance persistante. Parallèlement, les policiers (54\% d'entre eux) pensent que plus de $50 \%$ des jeunes délinquants sont des adolescents normaux et que pas plus de 3 à $10 \%$ ont des troubles mentaux ou affectifs graves. 
À cette évaluation plutôt réaliste du phénomène de la délinquance, les policiers proposent des mesures qui, pour $58 \%$ d'entre eux seraient un placement en internat pour 5 à $20 \%$ des jeunes délinquants et ils croient, $52 \%$ d'entre eux, que de 5 à $30 \%$ des mineurs délinquants peuvent pleinement profiter de la probation.

Finalement, notons (tableau 5, en annexe), que les policiers placent au premier rang des causes de la délinquance, le manque de surveillance de la part des parents, suivi d'une situation familiale malheureuse et en troisième lieu se retrouve le manque de respect envers l'autorité. Par la suite se regroupent sur un même pied d'égalité: la pauvreté, le désintéressement face aux études, le manque de débouchés d'emplois et trop de temps libre. À cet ordonnancement des sources de la délinquance des mineurs, les policiers donnent dans l'ordre, les facteurs suivants qui rendent difficile l'aide aux jeunes délinquants: ils ne veulent pas reconnaître leurs probèmes $(81 \%)$, ils ne veulent pas être aidés (75\%) et ils sont incapables de reconnaître leurs problèmes $(75 \%)$. Ces sources de difficuté dans l'intervention sont supportées par une mauvaise compréhension des procédures $(52 \%)$ et par des préjugés envers le système de justice $(62 \%)$.

L'ensemble de ces données nous portent à conclure que les policiers ont une perception tout à fait réaliste et conforme aux connaissances scientifiques des phénomènes de la délinquance et des difficultés que l'on rencontre dans l'action auprès des jeunes délinquants.

\section{Ils croient accomplir leurs tâches d'une façon adéquate}

Le traitement des jeunes contrevenants par la police s'observe plus directement lors de l'interrogatoire; la mesure de l'écart entre la pratique perçue, les attentes énoncées et les normes légales, est une bonne façon d'apprécier l'adéquacité de l'action des policiers. Le tableau 6 (en annexe) présente quelques données à cet égard.

Nous y notons un écart plutôt mince entre les pratiques et les attentes, ce qui a lieu actuellement et ce qui devrait avoir lieu. En effet $81 \%$ des policiers déclarent que l'interrogation d'un mineur a lieu, la plupart du temps, sans la présence d'un avocat et $64 \%$ pensent que cela devrait être ainsi; pour sa part, la Loi sur les jeunes contrevenants reconnaît à l'adolescent le droit à un avocat même à cette phase des procédures. L'interrogatoire en l'absence d'un adulte est plus rare, $54 \%$ des policiers disent que cela a lieu actuellement et l'écart avec ce qui devrait avoir lieu est plus restreint, $45 \%$ pensent 
que cela devrait être ainsi. Finalement la mise en garde au sujet des déclarations est courante, $74 \%$ disent le faire dans la plupart des cas et $83 \%$ disent que cela devrait avoir lieu. En somme, les pratiques sont adéquates par rapport à ce que les policiers pensent de ce qui devrait se faire et par rapport aux prescriptions légales en ce qui concerne les mineurs.

Si leurs pratiques d'interrogatoire sont adéquates, il faut quand bien même se demander dans quelle mesure les critères de décision auxquels ils donnent de l'importance, quand il s'agit d'intenter des procédures, sont ceux qui sont effectivement employés et généralement recommandés suivant les connaissances acquises sur le travail policier. Selon le tableau 7 (en annexe), les policiers attachent beaucoup d'importance à la qualité de la preuve $(81 \%)$ et au désir de la victime $(61 \%)$. Viennent ensuite, avec une importance modérée, l'âge $(89 \%)$, la gravité de l'infraction $(53 \%)$, les plans élaborés par le mineur pour s'amender $(52 \%)$ et les antécédents judiciaires $(46 \%)$. Ferment la marche l'attitude du jeune et celle des parents. L'ordre de ces critères reflète bien l'esprit de la loi, pensons plus particulièrement au jugement de la Cour suprême du Canada sur l'importance de la victime (Touchette-Bergeron), et ils sont congruents avec les recherches empiriques sur les décisions des policiers (voir, pour Montréal, LeBlanc, 1971, et en général, Gottfredson et Gottfredson, 1982).

En somme, nous avons identifié un modèle cohérent, rigoureux et légaliste d'action que les policiers valorisent et pratiquent.

\section{Et ils jugent le système de justice inefficace}

$70 \%$ des policiers pensent que l'actuelle Loi sur les jeunes délinquants est inadéquate pour traiter des cas des jeunes contrevenants. De plus, ils évaluent la qualité des services avec un ceil très critique. Ainsi (voir tableau 8, en annexe), ils ne donnent à aucun service une cote de bon ou très bon avec une majorité. Si $80 \%$ jugent adéquate les ressources relatives aux mesures de rechanges (déjudiciarisation), seulement de $\mathbf{4 0}$ à $50 \%$ d'entre eux déclarent adéquats quelques services: les foyers d'accueil, les foyers de groupe, le counseling familial et le counseling familial intensif. Les services qui apparaissent mauvais, même si aucun n'est très mauvais, sont les installations de détention avant et après décision et le counseling ou traitement contre la drogue et l'alcoolisme. Les autres services ont obtenu une cote entre mauvais et adéquat. 
Si la loi est inadéquate et la qualité des services plutôt pauvre, l'action du tribunal n'est pas meilleure car $77 \%$ des policiers pensent qu'il y a trop de cas par rapport au nombre de juges et $97 \%$ jugent le processus judiciaire trop lent pour être efficace. Cette perception plutôt négative, que les policiers nous renvoient de l'efficacité du système de justice pour mineurs, est confirmée par le fait que plus du quatre cinquième contre les délinquants dangereux, une meilleure dissuasion et plus de respect de la loi alors qu'environ les trois cinquièmes espèrent une amélioration de la réhabilitation.

\section{Tout en se sentant appuyés par la collectivité}

Les policiers interrogés pensent que notre système de justice est inadéquat et inefficace et ils proposeraient une attitude plus sévère. Ce en quoi ils se sentent pleinement appuyés par la collectivité, en effet $86 \%$ d'entre eux perçoivent leur communauté comme demandant moins d'indulgence et plus de sévérité à l'égard des mineurs délinquants. De plus, seulement $57 \%$ croient que leur communauté penche du côté de l'attribution d'une plus grande attention aux besoins de traitement des jeunes contrevenants et $74 \%$ d'entre eux perçoivent la collectivité comme indécise face à l'importance à donner aux droits des jeunes qui ont commis des délits.

\section{DES CHANGEMENTS DANS LA BONNE DIRECTION}

Cet article a analysé la position d'un échantillon représentatif de policiers de la C.U.M. appartenant à diverses catégories de personnel directement ou indirectement impliqué auprès des mineurs et expérimenté dans le travail policier et auprès des jeunes policiers adéquatement scolarisés et qui se présentent comme un groupe en pleine maturité. Au terme de notre analyse de leurs opinions sur la nouvelle Loi sur les jeunes contrevenants et de leur évaluation de l'actuel système de justice pour mineurs, il apparaît que les changements proposés par cette législation sont sur la bonne voie. Il ne faut plus seulement aider et protéger le mineur, il faut aussi le dissuader de commettre des actes délinquants et protéger la société contre les plus dangereux.

Si les policiers ont une opinion très réaliste du phénomène de la délinquance, il n'en demeure pas moins qu'ils favorisent un modèle de justice plutôt de type parens patriae, comme antérieurement; ils demandent plus de sévérité à l'égard des adolescents délinquants et ils se sentent, à tort ou à raison, pleinement supportés par la population dans cette attente. Il est apparu évident que l'option des poli- 
ciers, à savoir, accorder une dominante à l'objectif de la protection de la société, semble s'écarter de l'intention manifeste du législateur. Celui-ci favorise un niveau différent d'équilibre entre la protection de la société et l'aide aux jeunes. Cette option des policiers n'est pas surprenante mais elle nous interroge.

En effet, si l'on désirait réduire l'écart entre l'objectif manifeste du législateur et la position des policiers, et ici nous rejoignons l'objectif pédagogique de cet article, il y a des thèmes que l'on devrait aborder avec les policiers durant la phase préparatoire à la mise en application de la Loi sur les jeunes contrevenants: ces thèmes sont évidemment, la nature de l'équilibre que l'on désire maintenir entre les objectifs de protection de la société et d'aide aux jeunes, mais aussi faudrait-il mieux expliquer les options concernant entre autre l'âge maximal, les durées de sentence, la conservation des dossiers. De l'information sur les autres composantes du système de justice et de la communication avec les autres intervenants permettrait d'augmenter la compréhension que possèdent les policiers des problèmes et des résultats obtenus par les autres intervenants et de mieux leur faire apprécier la nature du travail qu'ils font.

Un tel processus de formation et d'information n'avait pas eu lieu au moment de cette enquête; il permettrait peut être de rapprocher les options des policiers des intentions des législateurs, mais on ne doit pas en attendre un impact significatif sur la façon dont ils remplissent leur tâche, car ils se conforment déjà très bien aux normes que la loi leur propose ou que la tradition leur impose. Sur le plan évaluatif, il nous semble que même si l'on peut augmenter la compréhension que les policiers possèdent des éléments de la Loi sur les jeunes contrevenants, il sera difficile de déplacer leur position fondamentale qui accorde une dominante manifeste à la protection de la société. La nature de leur tâche et la socialisation qu'elle implique sont des contraintes trop fortes que l'information ne pourra vaincre à court terme.

Il n'en demeure pas moins que les perceptions de la réalité, les façons de faire et les attitudes des policiers tendent vers l'équilibre recherché par le législateur. Ce n'est qu'en comparant les policiers, à d'autres intervenants, juges, procureurs de la couronne, avocats, agents de relations humaines de C.S.S., que l'on pourra véritablement apprécier leur position, leur ressemblance et leurs différences par rapport à tous les autres intervenants du système de justice pour mineurs. 


\section{TABLEAU 1}

Philosophie de la Loi sur les jeunes contrevenants

1. Les adolescents ne sauraient, dans tous les cas, être assimilés aux adultes quant à leur degré de responsabilité pour leurs actes illégaux.

$\begin{array}{cccccc}\begin{array}{c}\text { Tout à fait } \\ \text { d'accord }\end{array} & \text { D'accord } & \begin{array}{c}\text { Plutôt } \\ \text { d'accord }\end{array} & \begin{array}{c}\text { Plutôt en } \\ \text { desaccord }\end{array} & \begin{array}{c}\text { En } \\ \text { desaccord }\end{array} & \begin{array}{c}\text { Tout à foit } \\ \text { en desaccord }\end{array} \\ 21 \% & 27,2 \% & 17,4 \% & 14,9 \% & 14,9 \% & 4,6 \%\end{array}$

2. Les adolescents ne sauraient, dans tous les cas, être assimilés aux adultes, quant aux conséquences de leurs actes illégaux.

$\begin{array}{cccccc}\begin{array}{c}\text { Tout à fais } \\ \text { d'accord }\end{array} & \text { D'accord } & \begin{array}{c}\text { Plutôt } \\ \text { d'accord }\end{array} & \begin{array}{c}\text { Plusot en } \\ \text { désaccord }\end{array} & \begin{array}{c}\text { En } \\ \text { désaccord }\end{array} & \begin{array}{c}\text { Tous à fait } \\ \text { en désaccord }\end{array} \\ 15,4 \% & 26,2 \% & 15,4 \% & 17,4 \% & 20,5 \% & 5,1 \%\end{array}$

3. Les adolescents qui commettent des délits devraient être tenus responsables de leur conduite illicite.

\begin{tabular}{|c|c|c|c|c|c|}
\hline $\begin{array}{l}\text { Tout à fait } \\
\text { d'accord }\end{array}$ & D'aciord & $\begin{array}{c}\text { Phath } \\
\text { d'accord }\end{array}$ & $\begin{array}{l}\text { Plutot en } \\
\text { désaccord }\end{array}$ & $\begin{array}{c}\text { En } \\
\text { desactorat }\end{array}$ & $\begin{array}{l}\text { Thur a foit } \\
\text { en desaccort }\end{array}$ \\
\hline $42,6 \%$ & $23,6 \%$ & $22,7 \%$ & $2,6 \%$ & $2,1 \%$ & $1,5 \%$ \\
\hline
\end{tabular}

4. Lorsque les besoins de l'adolescent et la protection de la société ne peuvent être conciliés, la protection de la société doit primer.

\begin{tabular}{|c|c|c|c|c|c|}
\hline $\begin{array}{l}\text { Tout à fait } \\
\text { t'accord }\end{array}$ & D'accord & $\begin{array}{c}\text { fHutôt } \\
\text { d'uccort }\end{array}$ & $\begin{array}{l}\text { Plutot en } \\
\text { destitcord }\end{array}$ & $\begin{array}{c}\ln \\
\text { desetcord }\end{array}$ & $\begin{array}{l}\text { Foust à foit } \\
\text { en diesactort }\end{array}$ \\
\hline $39,5 \%$ & $19,5 \%$ & $22,6 \%$ & $10,3 \%$ & $5,1 \%$ & $3,1 \%$ \\
\hline
\end{tabular}

5. Les adolescents qui commettent des infractions ont des besoins speciaux en raison de leur état de dépendance et de leur degré de maturité.

$\begin{array}{cccccc}\begin{array}{c}\text { Tout à fait } \\ \text { d'accord }\end{array} & \text { v'accord } & \begin{array}{c}\text { Plutôt } \\ \text { d'accord }\end{array} & \begin{array}{c}\text { Plutôt en } \\ \text { désaccord }\end{array} & \begin{array}{c}\text { En } \\ \text { desaccord }\end{array} & \begin{array}{c}\text { Tour à fait } \\ \text { en desaccord }\end{array} \\ 14,9 \% & 30,8 \% & 27,70 \% & 10,3 \% & 11,3 \% & 5,1 \%\end{array}$

6. Les adolescents auxquels une infraction est imputée ont le droit de participer aux processus conduisant à des décisions qui les touchent.
frum a fait
d'accord
b'uceord
Phutit
phioten
En
desuccerd
Toust ù fait
$11,3 \%$
$25,6 \% \quad 26,70 \%$
$17,4 \%$
$13,8 \%$
$5,1 \%$

7. Les adolescents devraient avoir des garanties spéciales en ce qui concerne leurs droits et libertés.

$\begin{array}{cccccc}\begin{array}{c}\text { Tout à fait } \\ \text { d'accord }\end{array} & \text { D'accord } & \begin{array}{c}\text { Plutòt } \\ \text { d'accord }\end{array} & \begin{array}{c}\text { Plutôt en } \\ \text { désaccord }\end{array} & \begin{array}{c}\text { En } \\ \text { désaccord }\end{array} & \begin{array}{c}\text { Tout à fait } \\ \text { i’n désursort }\end{array} \\ 7,2 \% & 6,7 \% 0 & 16,9 \% & 13,8 \% & 26,7 \% & 28,70 \%\end{array}$

8. Dans leurs rapports avec le syst ème de justice pour les jeunes, les adolescents ont droit à ce qu'on leur applique le principe de l'intervention minimale.
Tout à fait
D'accord
Plutot
raccord
pturis en
désaciord
En
desaccord
Tout a fatt
$5,1 \%$
$23,6 \%$
$29,2 \%$
$18,5 \%$
$18,5 \%$
$5,1^{\mathrm{o} / 0}$

9. Les adolescents ne devraient être soustraits à l'autorité parentale que dans les seuls cas où les mesures comportant le maintien de cette autorité sont contreindiqués.

\begin{tabular}{cccccc}
$\begin{array}{c}\text { Tout à fait } \\
\text { d'accord }\end{array}$ & D'accord & $\begin{array}{c}\text { Plutôt } \\
\text { I'octord }\end{array}$ & $\begin{array}{c}\text { Phutôt en } \\
\text { désaccord }\end{array}$ & $\begin{array}{c}\text { En } \\
\text { désuccord }\end{array}$ & $\begin{array}{c}\text { Tout à fait } \\
\text { en désactord }\end{array}$ \\
$17,4 \%$ & $32,3 \%$ & $22,1 \%$ & $12,8 \%$ & $11,3 \%$ & $4,1 \%$ \\
\hline
\end{tabular}




\section{TABLEAU 2}

Mesures substantielles et procédurales prévues dans la Loi sur les jeunes contrevenants

\section{Mesures de rechange aux procédures judiciaires} (Mesures volontaires)

1. Le recours à des mesures de rechange (mesures volontaires) à l'endroit d'un adolescent à qui une infraction est imputée ne peut se faire que si l'adolescent, informé des mesures de rechange, a librement manifesté sa ferme volonté de collaborer à leur mise en cuvre.

$\begin{array}{cccccc}\begin{array}{c}\text { Tout a fait } \\ \text { d'accord }\end{array} & \text { D'accord } & \begin{array}{c}\text { Plutót } \\ \text { d'accord }^{\prime}\end{array} & \begin{array}{c}\text { Plutót en } \\ \text { désaccord }\end{array} & \begin{array}{c}\text { En } \\ \text { désaccord }\end{array} & \begin{array}{c}\text { Tout a fair } \\ \text { en désaccord }\end{array} \\ 30,3 \% & 32,3 \% & 10,3 \% & 6,7 \% & 10,3 \% & 10,3 \%\end{array}$

2. Le recours à des mesures de rechange (mesures volontaires) à l'endroit d'un adolescent à qui une infraction est imputée ne peut se faire que s'il y a des preuves suffisantes justifiant des poursuites relatives à l'infraction.

$\begin{array}{cccccc}\begin{array}{c}\text { Tout à fait } \\ \text { d'accord }\end{array} & \text { D'accord } & \begin{array}{c}\text { Plutôt } \\ \text { d'accord }\end{array} & \begin{array}{c}\text { Plutôt en } \\ \text { désaccord }\end{array} & \begin{array}{c}\text { En } \\ \text { desaccord }\end{array} & \begin{array}{c}\text { Tout d̀ fait } \\ \text { en désaccord }\end{array} \\ 22,1 \% & 36,9 \% & 16,4 \% & 9,2 \% & 11,8 \% & 3,6 \%\end{array}$

3. L'adolescent à qui une infraction est imputée ne peut faire l'objet de mesures de rechange (mesures volontaires) s'il dénie toute participation à la perpétration de l'infraction.

$\begin{array}{cccccc}\begin{array}{c}\text { Tout à fait } \\ \text { d'accord }\end{array} & \text { D'accord } & \begin{array}{c}\text { Plutôt } \\ \text { d'accord }\end{array} & \begin{array}{c}\text { Plutót en } \\ \text { désaccord }\end{array} & \begin{array}{c}\text { En } \\ \text { désaccord }\end{array} & \begin{array}{c}\text { Tout à fait } \\ \text { en desaccord }\end{array} \\ 25,6 \% & 22,6 \% & 9,2 \% & 11,8 \% & 14,9 \% & 15,9 \%\end{array}$

4. L'adolescent à qui une infraction est imputée ne peut faire l'objet de mesures de rechange (mesures volontaires) $s$ 'il manifeste le désir de voir déférer au tribunal pour adolescents (tribunal de la jeunesse) l'accusation portée contre lui.

$\begin{array}{cccccc}\begin{array}{c}\text { Tout à fait } \\ \text { d'accord }\end{array} & \text { D'accord } & \begin{array}{c}\text { Plutót } \\ \text { d'accord }\end{array} & \begin{array}{c}\text { Plutót en } \\ \text { désaccord }\end{array} & \begin{array}{c}\text { En } \\ \text { dessaccord }\end{array} & \begin{array}{c}\text { Tout à fait } \\ \text { en desaccord }\end{array} \\ 25,6 \% & 34,9 \% & 10,8 \% & 14,4 \% & 9,7 \% & 4,6 \%\end{array}$

5. Le recours aux mesures de rechange (mesures volontaires) à l'endroit d'un adolescent à qui une infraction est imputée n'empêche pas la mise en ceuvre de poursuites relatives à cette infraction.

$\begin{array}{cccccc}\begin{array}{c}\text { Tout à fait } \\ \text { d'accord }\end{array} & \text { D'accord } & \begin{array}{c}\text { Plutôt } \\ \text { d'accord }\end{array} & \begin{array}{c}\text { Plutót en } \\ \text { désaccord }\end{array} & \begin{array}{c}\text { En } \\ \text { desaccord }\end{array} & \begin{array}{c}\text { Tout à fait } \\ \text { en désaccord }\end{array} \\ 31,8 \% & 31,3 \% & 12,8 \% & 9,7 \% & 8,7 \% & 5,6 \%\end{array}$

6. Le juge doit rejeter les accusations portées contre un adolescent lorsque ce dernier a entièrement accompli les mesures de rechange prises à l'égard de l'infraction concernée.

$\begin{array}{cccccc}\begin{array}{c}\text { Tout à fait } \\ \text { d'accord }\end{array} & \text { D'accord } & \begin{array}{c}\text { Plutôt } \\ \text { d'accord }\end{array} & \begin{array}{c}\text { Plutôt en } \\ \text { désaccord }\end{array} & \begin{array}{c}\text { En } \\ \text { désaccord }\end{array} & \begin{array}{c}\text { Tout à fait } \\ \text { en désaccord }\end{array} \\ 12,8 \% & 18,5 \% & 16,9 \% & 14,9 \% & 20 \% & 16,9 \%\end{array}$

7. Le juge peut rejeter les accusations portées contre un adolescent lorsque ce dernier a partiellement accompli les mesures de rechange prises à l'égard de l'infraction concernée.

$\begin{array}{cccccc}\begin{array}{c}\text { Tout d fait } \\ \text { d'accord }\end{array} & \text { D'accord } & \begin{array}{c}\text { Plutat } \\ \text { d'accord }\end{array} & \begin{array}{c}\text { Plutôt en } \\ \text { désaccord }\end{array} & \begin{array}{c}\text { En } \\ \text { désaccord }\end{array} & \begin{array}{c}\text { Tout d fait } \\ \text { en desaccord }\end{array} \\ 3,6 \% & 7,2 \% & 6,7 \% & 19,9 \% & 31,8 \% & 31,3 \%\end{array}$


Mise en liberté sous caution et détention

8. Le tribunal pour adolescents traitera les demandes de mise en liberté sous caution selon les règles et critères établis à cet égard dans le code criminel.

$\begin{array}{cccccc}\begin{array}{c}\text { Tout à fait } \\ \text { d'accord }\end{array} & \text { D'accord } & \begin{array}{c}\text { Plutôt } \\ \text { d'accord }\end{array} & \begin{array}{c}\text { Plutot en } \\ \text { désaccord }\end{array} & \begin{array}{c}\text { En } \\ \text { désaccord }\end{array} & \begin{array}{c}\text { Tout d fait } \\ \text { en désaccord }\end{array} \\ 28,2 \% & 32,3 \% & 17,9 \% & 9,7 \% & 5,6 \% & 6,2 \%\end{array}$

9. Aucun adolescent ne peut être détenu dans un local en compagnie de délinquants adultes.

$\begin{array}{cccccc}\begin{array}{c}\text { Tout a fait } \\ \text { d'accord }\end{array} & \text { D'accord } & \begin{array}{c}\text { Plutôt } \\ \text { d'accord }\end{array} & \begin{array}{c}\text { Plutót en } \\ \text { desaccord }\end{array} & \begin{array}{c}\text { En } \\ \text { désaccord }\end{array} & \begin{array}{c}\text { Tout ò fait } \\ \text { en désaccord }\end{array} \\ 54,9 \% & 26,2 \% & 11,3 \% & 4,1 \% & 3,1 \% & 0,5 \%\end{array}$

Avis aux parents

10. Lorsqu'un adolescent est arrêté et détenu sous garde, il faut le plus tôt possible, aviser son père ou sa mère, ou un autre adulte approprié, de l'arrestation, de ses motifs et du lieu de détention.

$\begin{array}{cccccc}\begin{array}{c}\text { Tout à fait } \\ \text { d'accord }\end{array} & \text { D'accord } & \begin{array}{c}\text { Plutôt } \\ \text { d'accord }\end{array} & \begin{array}{c}\text { Plutôt en } \\ \text { désaccord }\end{array} & \begin{array}{c}\text { En } \\ \text { désaccord }\end{array} & \begin{array}{c}\text { Tout à fait } \\ \text { en désaccord }\end{array} \\ 56,4 \% & 32,8 \% & 7,2 \% & 1,5 \% & 1 \% & 1 \%\end{array}$

11. Lorsque l'avis n'a pas été donné et qu'aucune des personnes autorisées à le recevoir n'accompagne le jeune au tribunal le juge peut ajourner l'affaire et ordonner qu'un avis soit donné.

\begin{tabular}{|c|c|c|c|c|c|}
\hline $\begin{array}{l}\text { Tout a fait } \\
d \text { accord }\end{array}$ & D'accord & $\begin{array}{c}\text { Plusot } \\
\text { d'accord }\end{array}$ & $\begin{array}{l}\text { Plutôt en } \\
\text { déseccord }\end{array}$ & $\begin{array}{c}\text { En } \\
\text { desaccurd }\end{array}$ & $\begin{array}{l}\text { Iomt a fail } \\
\text { en siésactort }\end{array}$ \\
\hline $40,5 \%$ & $31,8 \%$ & $13,8 \%$ & $7,2 \%$ & $5,1 \%$ & $1,5 \%$ \\
\hline
\end{tabular}

12. Le juge peut dispenser de l'avis lorsque les circonstances l'exigent.

$\begin{array}{cccccc}\begin{array}{c}\text { Tout à fait } \\ \text { d'accord }\end{array} & \text { D'accord } & \begin{array}{c}\text { Plutôt } \\ d^{\prime} \text { 'occord }\end{array} & \begin{array}{c}\text { Plutồ en } \\ \text { désaccord }\end{array} & \begin{array}{c}\text { En } \\ \text { désaccord }\end{array} & \begin{array}{c}\text { Tout à fait } \\ \text { en désactord }\end{array} \\ 35,0 \% & 40,5 \% & 16,4 \% & 2,1 \% & 4,6 \% & 0,5 \%\end{array}$

13. Le défaut de donner l'avis au père ou à la mère, ou à un autre adulte approprié rend invalides les procédures engagées sous le régime de la Loi sur les jeunes contrevenants, à moins que le tribunal n'ait dispensé de l'avis.
Tout à fait
d'accord
Doccord
Plutôt
Pitutât en
En
désaccord
Tout a fait
$10,8 \%$
$22,6 \%$
$12,3 \%$
$12,3 \%$
$22,6 \%$
$14,5 \%$

14. Le défaut de donner l'avis au père ou à la mère, ou à un autre adulte approprié rend invalides les procédures engagées sous le régime de la Loi sur les jeunes contrevenants, à moins que le père ou la mère, de sa propre initiative, ne soit présent au tribunal.

$\begin{array}{cccccc}\begin{array}{c}\text { Tout à foit } \\ \text { d'accord }\end{array} & \text { D'accord } & \begin{array}{c}\text { Plutôt } \\ \text { d'accord }\end{array} & \begin{array}{c}\text { Plutôt en } \\ \text { désaccord }\end{array} & \begin{array}{c}\text { En } \\ \text { désaccord }\end{array} & \begin{array}{c}\text { Tout à fait } \\ \text { en désaccord }\end{array} \\ 6,7 \% & 20,5 \% & 11,8 \% & 12,3 \% & 26,2 \% & 22,6 \%\end{array}$

\section{Services d'un avocat}

15. L'adolescent a le droit d'obtenir sans retard les services d'un avocat à toute phase des poursuites intentées contre lui.
Tout d foit
d'accord
$D$ 'accord
Plutôt
Plutôt en
En
désaccord
Tout à fait
$22,6 \%$
$36,9 \%$
$13,8 \%$
$7,7 \%$
$11,3 \%$
en desaccord
$7,7 \%$ 
16. L'adolescent arrêté doit être informé par la police de son droit aux services d'un avocat, et on doit lui donner l'occasion d'exercer ce droit.

$\begin{array}{cccccc}\begin{array}{c}\text { Tout à fait } \\ \text { d'accord }\end{array} & \text { D'accord } & \begin{array}{c}\text { Plutot } \\ \text { d'accord }\end{array} & \begin{array}{c}\text { Plutôt en } \\ \text { désaccord }\end{array} & \begin{array}{c}\text { En } \\ \text { desaccord }\end{array} & \begin{array}{c}\text { Tout à fait } \\ \text { en désaccord }\end{array} \\ 20 \% & 44,1 \% & 10,8 \% & 8,2 \% & 10,3 \% & 6,7 \%\end{array}$

17. L'adolescent qui $n$ 'est pas représenté par un avocat à une audience du tribunal pour adolescents (tribunal de la jeunesse) doit être informé de son droit d'être représenté par un avocat et on doit lui fournir l'occasion d'exercer ce droit.

$\begin{array}{cccccc}\begin{array}{c}\text { Tout d fait } \\ \text { d'accord }\end{array} & \text { D'accord } & \begin{array}{c}\text { Plutôt } \\ \text { d'accord }\end{array} & \begin{array}{c}\text { Plutót en } \\ \text { désaccord }\end{array} & \begin{array}{c}\text { En } \\ \text { désaccord }\end{array} & \begin{array}{c}\text { Tout d fait } \\ \text { en désaccord }\end{array} \\ 28,7 \% & 42,6 \% & 13,3 \% & 3,6 \% & 6,7 \% & 5,1 \%\end{array}$

18. Lorsqu'il semble y avoir conflit entre les intérêts de l'adolescent et ceux de ses père ou mère, le juge doit s'assurer que l'adolescent est représenté par un avocat n'ayant aucun lien avec ces derniers.

$\begin{array}{cccccc}\begin{array}{c}\text { Tout à fait } \\ \text { d'accord }\end{array} & \text { D'accord } & \begin{array}{c}\text { Plutot } \\ \text { d'accord }\end{array} & \begin{array}{c}\text { Plutót en } \\ \text { désaccord }\end{array} & \begin{array}{c}\text { En } \\ \text { desaccord }\end{array} & \begin{array}{c}\text { Tout da fait } \\ \text { en désaccord }\end{array} \\ 26,7 \% & 43,6 \% & 14,4 \% & 5,6 \% & 5,1 \% & 4,6 \%\end{array}$

19. L'adolescent qui n'est pas représenté par un avocat à une audience du tribunal pour adolescents (tribunal de la jeunesse) peut, s'il en fait la demande, se faire assister par un adulte que le tribunal considère convenable.

$\begin{array}{cccccc}\begin{array}{c}\text { Tout à fait } \\ \text { d'accord }\end{array} & \text { D'accord } & \begin{array}{c}\text { P'utôt } \\ \text { d'accord }\end{array} & \begin{array}{c}\text { Plutôt en } \\ \text { désaccord }\end{array} & \begin{array}{c}\text { En } \\ \text { désaccord }\end{array} & \begin{array}{c}\text { Tout à fait } \\ \text { en désaccord }\end{array} \\ 20,5 \% & 39,5 \% & 16,9 \% & 5,6 \% & 11,3 \% & 6,2 \%\end{array}$

\section{Empreintes digitales et photographies}

20. Il est permis de relever les empreintes digitales ou de prendre la photographie d'un adolescent lors d'un acte criminel en tout cas où un adulte peut être soumis à ces procédures.

$\begin{array}{cccccc}\begin{array}{c}\text { Tout à fait } \\ \text { d'accord }\end{array} & \text { D'accord } & \begin{array}{c}\text { Plutôt } \\ d^{\prime} a c c o r d\end{array} & \begin{array}{c}\text { Plutôt en } \\ \text { désaccord }\end{array} & \begin{array}{c}\text { En } \\ \text { désaccord }\end{array} & \begin{array}{c}\text { Tour à fait } \\ \text { en desaccord }\end{array} \\ 48, \% & 22,6 \% & 12,8 \% & 3,6 \% & 6,2 \% & 6,7 \%\end{array}$

21. Il n'est pas permis de relever les empreintes digitales d'un adolescent lors d'une infraction punissable sur déclaration sommaire de culpabilité, même avec le consentement de celui-ci.

$\begin{array}{cccccc}\begin{array}{c}\text { Tout d fait } \\ \text { d'accord }\end{array} & \text { D'accord } & \begin{array}{c}\text { Plutot } \\ \text { d'accord }\end{array} & \begin{array}{c}\text { Plutôt en } \\ \text { désaccord }\end{array} & \begin{array}{c}\text { En } \\ \text { désaccord }\end{array} & \begin{array}{c}\text { Tout ò fait } \\ \text { en désaccord }\end{array} \\ 16,9 \% & 27,2 \% & 16,9 \% & 13,3 \% & 13,8 \% & 11,8 \%\end{array}$

\section{Décisions}

22. Les décisions prononcées à l'endroit d'un adolescent ne doivent en aucun cas aboutir à une peine plus grave que la peine maximale dont est passible l'adulte qui commet la même infraction.

$\begin{array}{cccccc}\begin{array}{c}\text { Tout d fait } \\ \text { d'accord }\end{array} & \text { D'accord } & \begin{array}{c}\text { Plutôt } \\ \text { d'accord }\end{array} & \begin{array}{c}\text { Plutot en } \\ \text { désaccord }\end{array} & \begin{array}{c}\text { En } \\ \text { desaccord }\end{array} & \begin{array}{c}\text { Tout d foit } \\ \text { en désaccord }\end{array} \\ 67,2 \% & 26,7 \% & 2,6 \% & 1,5 \% & 1 \% & 1 \%\end{array}$

23. Un jeune contrevenant peut s'acquitter d'une amende imposée par le tribunal pour adolescents (Tribunal de la jeunesse) en effectuant des travaux dans le cadre d'un programme établi à cette fin par la province.

$\begin{array}{cccccc}\begin{array}{c}\text { Tout d fair } \\ \text { d'accord }\end{array} & \text { D'accord } & \begin{array}{c}\text { Plutôt } \\ \text { d'accord }\end{array} & \begin{array}{c}\text { Plutôt en } \\ \text { désaccord }\end{array} & \begin{array}{c}\text { En } \\ \text { désaccord }\end{array} & \begin{array}{c}\text { Tout d foit } \\ \text { en désaccord }\end{array} \\ 49,7 \% & 35,9 \% & 11,3 \% & 1,5 \% & 0,5 \% & 1 \%\end{array}$


24. Sur consentement de l'adolescent et de ses parents, un juge du tribunal pour adolescents (tribunal de la jeunesse) peut rendre une décision exigeant la détention de cet adolescent pour le traitement d'un problème psychiatrique, psychologique ou médical.

$\begin{array}{cccccc}\begin{array}{c}\text { Tout à fait } \\ \text { d'accord }\end{array} & \text { D'accord } & \begin{array}{c}\text { Plutot } \\ \text { d'accord }\end{array} & \begin{array}{c}\text { Plutsiten } \\ \text { désaccord }\end{array} & \begin{array}{c}\text { En } \\ \text { désaccord }\end{array} & \begin{array}{c}\text { Tout à fait } \\ \text { en désaccord }\end{array} \\ 61,5 \% & 26,7 \% & 4,6 \% & 2,6 \% & 3,6 \% & 1 \%\end{array}$

25. Le tribunal pour adolescents (tribunal de la jeunesse), plutôt que les autorités provinciales, assume la responsabilité de déterminer si le niveau approprié du placement sous garde d'un jeune contrevenant, sera sécuritaire ou non sécuritajre.

$\begin{array}{cccccc}\begin{array}{c}\text { Tout à fait } \\ \text { d'accord }\end{array} & \text { D'accord } & \begin{array}{c}\text { Plutôt } \\ \text { d'accord }\end{array} & \begin{array}{c}\text { Plutôt en } \\ \text { désaccord }\end{array} & \begin{array}{c}\text { En } \\ \text { désaccord }\end{array} & \begin{array}{c}\text { Tout d fait } \\ \text { en désaccord }\end{array} \\ 30,3 \% & 45,1 \% & 12,8 \% & 6,2 \% & 4,6 \% & 1 \%\end{array}$

26. Les autorités provinciales, plutôt que le tribunal pour adolescents (tribunal de la jeunesse), auront la responsabilité de désigner l'endroit approprié du placement sous garde d'un jeune contrevenant, compte tenu du niveau de sécurité déterminé par le tribunal).

$\begin{array}{cccccc}\begin{array}{c}\text { Tout a fait } \\ \text { d'accord }\end{array} & \text { D'accord } & \begin{array}{c}\text { Plutôt } \\ \text { d'accord }\end{array} & \begin{array}{c}\text { Plutot en } \\ \text { désaccord }\end{array} & \begin{array}{c}\text { En } \\ \text { désaccord }\end{array} & \begin{array}{c}\text { Tout à fait } \\ \text { en désaccord }\end{array} \\ 10,3 \% & 23,1 \% 0 & 11,8 \% & 15,9 \% & 26,7 \% & 12,3 \%\end{array}$

\section{Examen (révision) des décisions}

27. Une commission provinciale peut être mise sur pied afin d'examiner les décisions comportant un placement sous garde.

$\begin{array}{cccccc}\begin{array}{c}\text { Tout a fait } \\ \text { d'accord }\end{array} & \text { D'accord } & \begin{array}{c}\text { Plutôt } \\ \text { d'accord }\end{array} & \begin{array}{c}\text { Plutôt en } \\ \text { désaccord }\end{array} & \begin{array}{c}\text { En } \\ \text { désaccord }\end{array} & \begin{array}{c}\text { Tout d fait } \\ \text { en désaccort }\end{array} \\ 10,8 \% & 27,2 \% & 16,4 \% & 15,4 \% & 17,9 \% & 12,3 \%\end{array}$

28. Le tribunal pour adolescents (tribunal de la jeunesse) conserve juridiction pour réexaminer toute décision jusqu'à ce que celle-ci soit complétée.

$\begin{array}{cccccc}\begin{array}{c}\text { Tout à fait } \\ \text { d'accord }\end{array} & \text { D'accord } & \begin{array}{c}\text { Plutôt } \\ \text { d'accord }\end{array} & \begin{array}{c}\text { Plutôt en } \\ \text { désactord }\end{array} & \begin{array}{c}\text { En } \\ \text { désaccord }\end{array} & \begin{array}{c}\text { Tout à fait } \\ \text { en désaccord }\end{array} \\ 23,6 \% & 54,4 \% & 13,3 \% & 3,6 \% & 3,1 \% & 2,1 \%\end{array}$

29. L'adolescent qui a été placé sous garde pendant un an doit être amené devant le tribunal pour adolescents (tribunal de la jeunesse) afin que ce dernier réexamine sa décision.

$\begin{array}{cccccc}\begin{array}{c}\text { Tout à fait } \\ \text { d'accord }\end{array} & \text { D'accord } & \begin{array}{c}\text { Plutôt } \\ \text { d'accord }^{\prime}\end{array} & \begin{array}{c}\text { Plutôt en } \\ \text { désaccord }\end{array} & \begin{array}{c}\text { En } \\ \text { désaccord }\end{array} & \begin{array}{c}\text { Tout à fait } \\ \text { en désaccord }\end{array} \\ 24,10 \% & 42,7 \% & 19 \% & 3,6 \% & 2,6 \% & 3,1 \%\end{array}$

30. Une jeune contrevenant peut demander à un juge du tribunal pour adolescents (tribunal de la jeunesse) de réexaminer toute décision qui ne comporte pas un placement sous garde.

$\begin{array}{cccccc}\begin{array}{c}\text { Tout d fait } \\ \text { d'accord }\end{array} & \text { D'accord } & \begin{array}{c}\text { Plutôt } \\ \text { d'accord }\end{array} & \begin{array}{c}\text { Plutót en } \\ \text { désaccord }\end{array} & \begin{array}{c}\text { En } \\ \text { desaccord }\end{array} & \begin{array}{c}\text { Tout à fait } \\ \text { en désaccord }\end{array} \\ 11,3 \% & 34,9 \% & 22,6 \% & 13,3 \% & 13,3 \% & 4,6 \%\end{array}$

31. Les parents d'un jeune contrevenant peuvent demander à un juge du tribunal pour adolescents (tribunal de la jeunesse) de réexaminer toute décision qui ne comporte pas un placement sous garde.

$\begin{array}{cccccc}\begin{array}{c}\text { Tout a fait } \\ \text { d'accord }\end{array} & \text { D'accord } & \begin{array}{c}\text { Plutôt } \\ \text { d'accord }\end{array} & \begin{array}{c}\text { Plutot en } \\ \text { désaccord }\end{array} & \begin{array}{c}\text { En } \\ \text { désaccord }\end{array} & \begin{array}{c}\text { Tout à fait } \\ \text { en désaccord }\end{array} \\ 10,3 \% & 40,5 \% & 27,2 \% & 11,8 \% & 7,2 \% & 3,1 \%\end{array}$


32. Les autorités provinciales peuvent demander à un juge du tribunal pour adolescents (tribunal de la jeunesse) de réexaminer toute décision qui ne comporte pas un placement sous garde.

$\begin{array}{cccccc}\begin{array}{c}\text { Tout ò fait } \\ \text { d'accord }\end{array} & \text { D'accond } & \begin{array}{c}\text { Plutôt } \\ \text { d'accord }\end{array} & \begin{array}{c}\text { Plutốt en } \\ \text { désaccord }\end{array} & \begin{array}{c}\text { En } \\ \text { desaccord }\end{array} & \begin{array}{c}\text { Tout à fait } \\ \text { en désaccord }\end{array} \\ \mathbf{8 , 7 \%} & 31,8 \% & 20,5 \% & 16,4 \% & 13,8 \% & 9,2 \%\end{array}$

33. Le procureur de la couronne peut demander qu'un juge du tribunal pour adolescents (tribunal de la jeunesse) réexamine toute décision qui ne comporte pas un placement sous garde.

$\begin{array}{cccccc}\begin{array}{c}\text { Tour à fait } \\ \text { d'accord }\end{array} & \text { D'accord } & \begin{array}{c}\text { Plutôt } \\ \text { d'accord }\end{array} & \begin{array}{c}\text { Plutôt en } \\ \text { désaccord }\end{array} & \begin{array}{c}\text { En } \\ \text { desaccord }\end{array} & \begin{array}{c}\text { Tout à fait } \\ \text { en désaccord }\end{array} \\ 19,5 \% & 41,5 \% & 22,1 \% & 8,2 \% & 6,2 \% & 2,6 \%\end{array}$

\section{APPEL}

34. La Loi sur les jeunes contrevenants accorde aux adolescents des droits d'appel similaires à ceux dont jouissent les adultes en vertu du Code criminel.

$\begin{array}{cccccc}\begin{array}{c}\text { Tout ò fait } \\ \text { d'accord }\end{array} & \text { D'accord } & \begin{array}{c}\text { Plutôt } \\ \text { d'accord }\end{array} & \begin{array}{c}\text { Plutôt en } \\ \text { desaccord }\end{array} & \begin{array}{c}\text { En } \\ \text { desaccord }\end{array} & \begin{array}{c}\text { Tout à fait } \\ \text { en desaccord }\end{array} \\ 21,5 \_ & 32,8 \% & 20,5 \% & 10,3 \% & 10,8 \% & 4,1 \%\end{array}$

\section{Audiences publiques}

35. En général, les audiences tenues en vertu de la Loi sur les jeunes contrevenants seront publiques.

$\begin{array}{cccccc}\begin{array}{c}\text { Tout d fait } \\ \text { d'accord }\end{array} & \text { D'accord } & \begin{array}{c}\text { Plutôt } \\ \text { d'accord }\end{array} & \begin{array}{c}\text { Plutôt en } \\ \text { désnccord }\end{array} & \begin{array}{c}\text { En } \\ \text { désaccond }\end{array} & \begin{array}{c}\text { Tout à fait } \\ \text { en désaccord }\end{array} \\ 16,4 \% & 14,9 \% & 8,2 \% & 18,5 \% & 23,1 \% & 19 \%\end{array}$

36. Dans certaines circonstances, le juge peut exclure certains assistants ou toute l'assistance de la salle d'audience.

$\begin{array}{cccccc}\begin{array}{c}\text { Tout à fait } \\ \text { d'accord }\end{array} & \text { D'accord } & \begin{array}{c}\text { Plutôt } \\ \text { d'accord }\end{array} & \begin{array}{c}\text { Plưôt en } \\ \text { désaccord }\end{array} & \begin{array}{c}\text { En } \\ \text { desaccord }\end{array} & \begin{array}{c}\text { Tout à fait } \\ \text { en désaccord }\end{array} \\ 45,6 \% & 39,5 \% & 7,7 \% & 1,5 \% & 2,6 \% & 3,1 \%\end{array}$

\section{Communication des dossiers}

37. Normalement, tous les documents faisant partie d'un dossier du tribunal pour adolescents doivent être mis à la disposition de l'adolescent concerné, à sa demande.

$\begin{array}{cccccc}\begin{array}{c}\text { Towt d fait } \\ \text { d'accord }\end{array} & \text { D'accord } & \begin{array}{c}\text { Plutôt } \\ \text { d'accord }\end{array} & \begin{array}{c}\text { Plutôt en } \\ \text { désaccord }\end{array} & \begin{array}{c}\text { En } \\ \text { desaccord }\end{array} & \begin{array}{c}\text { Tout à fait } \\ \text { en désaccord }\end{array} \\ 7,2 \% & 12,3 \% & 15,4 \% & 23,6 \% & 22,6 \% & 19 \%\end{array}$

38. Le tribunal peut empêcher que l'adolescent n'ait accès à certains rapports faisant partie du dossier du tribunal si les informations qu'ils contiennent sont de nature à entraver le traitement ou la guérison de l'adolescent en question.

$\begin{array}{cccccc}\begin{array}{c}\text { Tout à fait } \\ \text { d'accord }\end{array} & \text { D'accord } & \begin{array}{c}\text { Plutôt } \\ \text { d'accord }\end{array} & \begin{array}{c}\text { Plutót en } \\ \text { désaccord }\end{array} & \begin{array}{c}\text { En } \\ \text { désaccord }\end{array} & \begin{array}{c}\text { Tout à fait } \\ \text { en désaccord }\end{array} \\ 51,3 \% & 36,9 \% & 8,7 \% & 1,5 \% & 0,5 \% & 1 \%\end{array}$

39. Le tribunal peut empêcher que l'adolescent n'ait accès à certains rapports faisant partie du dossier du tribunal si les informations qu'ils contiennent sont de nature à causer un préjudice physique ou mental à un tiers.

$\begin{array}{cccccc}\begin{array}{c}\text { Tout dे fait } \\ \text { d'accord }\end{array} & \text { D'accord } & \begin{array}{c}\text { Plutot } \\ \text { d'accord }\end{array} & \begin{array}{c}\text { Plutót en } \\ \text { désaccord }\end{array} & \begin{array}{c}\text { En } \\ \text { desaccord }\end{array} & \begin{array}{c}\text { Tout à fait } \\ \text { en desaccord }\end{array} \\ 60 \% & 31,3 \% & 6,2 \% & 10 \% & 0,5 \% & 1 \%\end{array}$




\section{Destruction des dossiers}

A. En vertu de la Loi sur les jeunes contrevenants, un contrevenant dont le dossier est constitué uniquement d'infractions punissables sur déclaration sommaire de culpabilité et qui n'a commis aucune autre infraction dans les deux ans qui suivent l'exécution complète de toute décision dont il avait fait l'objet, verra son dossier du tribunal détruit.

a) Â notre avis, ce dossier devrait-il être éventuellement détruit dans les cas d'infractions punissables sur déclaration sommaire de culpabilité?
1. $(34,2 \%)$
2. $(64$,
$64,1 \%)$ NON
3. $(4,6 \%)$ NE SAIS PAS

b) DANS L'AFFIRMATIVE: après l'exécution complète de la décision dont l'adolescent a fait l'objet, pendant combien de temps ces dossiers devraient-ils être conservés avant d'être détruits?
1. $(44,6 \%) 2$ ans 2 . $(28,1 \%) 5$ ans $3 .(27,3 \%)$ autres

B. En vertu de la Loi sur les jeunes contrevenants, un contrevenant qui a complété sa sentence pour un acte criminel et qui n'a commis aucune autre infraction dans les cinq ans qui suivent l'exécution complète de toute décision dont il avait fait l'objet verra son dossier du tribunal détruit.

a) Ả votre avis, ce dossier devrait-il être éventuellement détruit dans les cas d'actes criminels?

1. $(50,8 \%)$ OUI 2. $(45,6 \%)$ NON 3. $(3,6 \%)$ NE SAIS PAS

b) DANS L'AFFIRMATIVE: Après l'exécution complète de la décision dont l'adolescent a fait l'objet, pendant combien de temps ces dossiers devaient-ils être conservés avant d'être détruits?

1. $(27,2)$ Moins de 5 ans $2 .(64,1 \%) 5$ ans $3 .(8,7 \%)$ plus de 5 ans

\section{TABLEAU 3}

La gravité de la delinquance dans la communauté

\begin{tabular}{lrccc}
\hline & $1^{\text {er }}$ choix & $2^{\text {e }}$ choix & $3^{e}$ choix & $4^{e}$ choix \\
\hline $\begin{array}{l}\text { Violence } \\
\text { en général } \\
\text { homicide }\end{array}$ & 5,3 & 19,2 & 9,4 & 11,4 \\
$\begin{array}{l}\text { vol à main armé } \\
\text { contre les biens } \\
\quad \begin{array}{l}\text { en général } \\
\text { vol par effraction }\end{array}\end{array}$ & 51,6 & 17,6 & 8,8 & 4,5 \\
$\begin{array}{l}\text { autres (drogue, } \\
\text { prostitution) }\end{array}$ & 6,5 & 17,9 & 49,2 & 60,2 \\
\hline
\end{tabular}




\section{TABLEAU 4}

Parmi les jeunes de votre région à l'égard desquels la police recommande d'intenter des procedures, combien sont:

\begin{tabular}{rrcccc}
\hline$\%$ & $\begin{array}{c}\text { Une menace } \\
\text { retle pour } \\
\text { la societe }\end{array}$ & $\begin{array}{c}\text { Des enfants } \\
\text { normaux }\end{array}$ & $\begin{array}{c}\text { Ont des } \\
\text { troubles } \\
\text { affectifs } \\
\text { graves }\end{array}$ & $\begin{array}{c}\text { Devraient } \\
\text { stre placts } \\
\text { en internat }\end{array}$ & $\begin{array}{c}\text { Peuvent } \\
\text { profiter de } \\
\text { la probation }\end{array}$ \\
\hline 1 & & 1,7 & 4,1 & 2,3 & 1,7 \\
2 & 0,6 & 0,6 & 5,2 & 2,8 & 1,7 \\
3 & 1,7 & 0,6 & 10,5 & 5,6 & 2,3 \\
5 & 10,1 & 7,5 & 15,7 & 16,9 & 9,7 \\
10 & 14,5 & 9,2 & 21,5 & 22,6 & 13,7 \\
20 & 13,4 & 7,5 & 9,9 & 18,6 & 16,0 \\
30 & 12,8 & 8,1 & 9,3 & 10,7 & 12,6 \\
40 & 7,8 & 4,0 & 4,7 & 3,4 & 6,3 \\
50 & 10,6 & 9,8 & 7,6 & 9,6 & 16,0 \\
60 & 6,1 & 15,6 & 3,5 & 3,4 & 5,7 \\
70 & 8,4 & 15,6 & 1,7 & 0,6 & 6,3 \\
80 & 6,7 & 13,3 & 1,7 & 1,1 & 4,0 \\
90 & 6,1 & 5,8 & 4,1 & 1,1 & 3,4 \\
100 & 2,2 & 0,6 & 0,6 & 1,1 & 0,6 \\
\hline
\end{tabular}

\section{TABLEAU 5}

$A$ votre avis, dans quelle mesure chacun des facteurs suivants contribue-t-il à la délinquance? Veuillez encercler le chiffre approprié à chaque mention

\begin{tabular}{|c|c|c|c|c|c|}
\hline & $\begin{array}{c}\text { Extra- } \\
\text { mement }\end{array}$ & Beaucoup & $\begin{array}{l}\text { Modé- } \\
\text { rément }\end{array}$ & Peu & $\begin{array}{c}\text { Pas } \\
\text { du tout }\end{array}$ \\
\hline La pauvreté & 12,8 & 40 & 28,7 & 17,4 & 0,5 \\
\hline $\begin{array}{l}\text { Les pressions des pairs } \\
\text { Le manque de surveillance de }\end{array}$ & 11,3 & 43,1 & 31,3 & 8,2 & 0,5 \\
\hline $\begin{array}{l}\text { la part des parents } \\
\text { Une situation familiale }\end{array}$ & 47,7 & 43,1 & 7,7 & 0,5 & 0,5 \\
\hline malheureuse & 33,8 & 49,2 & 14,4 & 1,5 & 1 \\
\hline $\begin{array}{l}\text { Trop de temps libre } \\
\text { Le manque de respect envers } \\
\text { l'autorité (parents, profes- } \\
\text { seurs, police, etc.) }\end{array}$ & 14,9 & 37,9 & 32,3 & 13,8 & 1 \\
\hline $\begin{array}{l}\text { Le désintéressement des } \\
\text { enfants à l'égard des études } \\
\text { Le manque de débouchés }\end{array}$ & 10,8 & 39,5 & 38,5 & 9,7 & 1,5 \\
\hline $\begin{array}{l}\text { d'emplois pour les jeunes } \\
\text { La stigmatisation à la suite } \\
\text { d'un premier contact avec } \\
\text { le système de justice pour } \\
\text { les jeunes }\end{array}$ & 12,3 & 33,8 & 36,4 & 15,9 & 10,3 \\
\hline
\end{tabular}




\section{TABLEAU 6}

Le traitement des jeunes contrevenants par la police

A. L'interrogatoire, en l'absence d'un avocat, d'un jeune placé sous garde, est une pratique qui:

$\begin{array}{cccccc}\text { Dans tous Dansla } & \text { Dansla Dans En aucun } & \text { Ne sais } \\ \text { les cas plupart } & \text { moitié quelques cas } & \text { pas } \\ & \text { des cas } & \text { descas cas } & & \\ \text { environ }\end{array}$

1. a lieu actuel-

$\begin{array}{lcccccc}\begin{array}{l}\text { ment } \\ \text { devrait avoir }\end{array} & 20,5 & 61 & 4,6 & 9,7 & 3,6 & 0,5 \\ \text { lieu } & 18,5 & 45,1 & 6,2 & 23,6 & 5,6 & 1\end{array}$

B. L'interrogatoire, en l'absence d'un adulte (père, mère, tuteur ou membre de la parenté), d'un jeune placé sous garde, est une pratique qui:

$\begin{array}{ccccc}\text { Dans tous Dansla } & \text { Dans la Dans En aucun } & \text { Ne sais } \\ \text { les cas plupart } & \text { moitié quelques cas } & \text { pas } \\ \text { des cas des cas cas } & & \\ \text { environ } & & \end{array}$

1. a lieu actuel-

$\begin{array}{lllllll}\text { ment } & 5,6 & 48,2 & 16,4 & 25,6 & 3,6 & 0,5\end{array}$

2. devrait avoir $\begin{array}{llllll}\text { lieu } & 10,8 & 36,4 & 10,8 & 29,7 & 12,3\end{array}$

C. La mise en garde des jeunes en ce qui concerne les déclarations qu'ils pourraient faire à propos d'une infraction alléguée est une pratique qui:

$\begin{array}{ccccc}\text { Dans tous Dans la } & \text { Dansla Dans En aucun } & \text { Ne sais } \\ \text { les cas plupart } & \text { moitié quelques cas } & \text { pas } \\ \text { descas des cas cas } & & \\ \text { environ }\end{array}$

1. a lieu actuel$\begin{array}{lllllll}\text { ment } & 33,3 & 40,5 & 7,2 & 15,9 & 1,5 & 1,5\end{array}$

2. devrait avoir lieu

\begin{tabular}{rrrrrr}
55,4 & 28,2 & 3,6 & 5,1 & 7,2 & 0,5 \\
\hline
\end{tabular}




\section{TABLEAU 7}

Plusieurs facteurs entrent en ligne de compte dans la décision soit de recommander des procédures a l'endroit d'un jeune que vous soupconnez d'avoir commis une infraction, soit de simplement lui donner un avertissement. Veuillez indiquer l'importance que vous accordez à chacun des facteurs suivants dans cette décision, en encerclant le chiffre approprié

\begin{tabular}{|c|c|c|c|c|c|}
\hline & & $\begin{array}{c}\text { Aucune } \\
\text { importance }\end{array}$ & $\begin{array}{c}\text { Peu } \\
\text { d'importance }\end{array}$ & $\begin{array}{l}\text { Importance } \\
\text { moderte }\end{array}$ & $\begin{array}{l}\text { Beaucoup } \\
\text { d'importance }\end{array}$ \\
\hline a) & $\begin{array}{l}\text { Les preuves à fournir } \\
\text { pour porter l'affaire }\end{array}$ & & & & \\
\hline b) & $\begin{array}{l}\text { devant le tribunal } \\
\text { La gravité de }\end{array}$ & & 1 & 17,8 & 81,2 \\
\hline & l'infraction & 1,6 & 9,9 & 53,2 & 34,9 \\
\hline c) & $\begin{array}{l}\text { Les plans élaborés par } \\
\text { le jeune pour s'amender }\end{array}$ & 2,1 & 16,1 & 51,6 & 30,2 \\
\hline d) & $\begin{array}{l}\text { La conduite, par ex. } \\
\text { respectueuse ou rebelle, } \\
\text { du jeune }\end{array}$ & 19,5 & 37,4 & 31,3 & 11,8 \\
\hline e) & Les désirs de la victime & & 3,6 & 35,4 & 61 \\
\hline f) & $\begin{array}{l}\text { Les antécédents } \\
\text { judiciaires du jeune } \\
\text { L'attitude des parents }\end{array}$ & 3,1 & 14,9 & 46,2 & 35,9 \\
\hline & ou du tuteur & 1,5 & 15,9 & 33,8 & 48,7 \\
\hline h) & L'âge du jeune & 0,5 & 0,5 & 89,2 & 9,7 \\
\hline
\end{tabular}




\section{TABLEAU 8}

Comment évalueriez-vous la qualité des services suivants offerts aux adolescents de votre collectivité. Veuillez encercler le chiffre qui correspond à votre évaluation de chacun de ces services

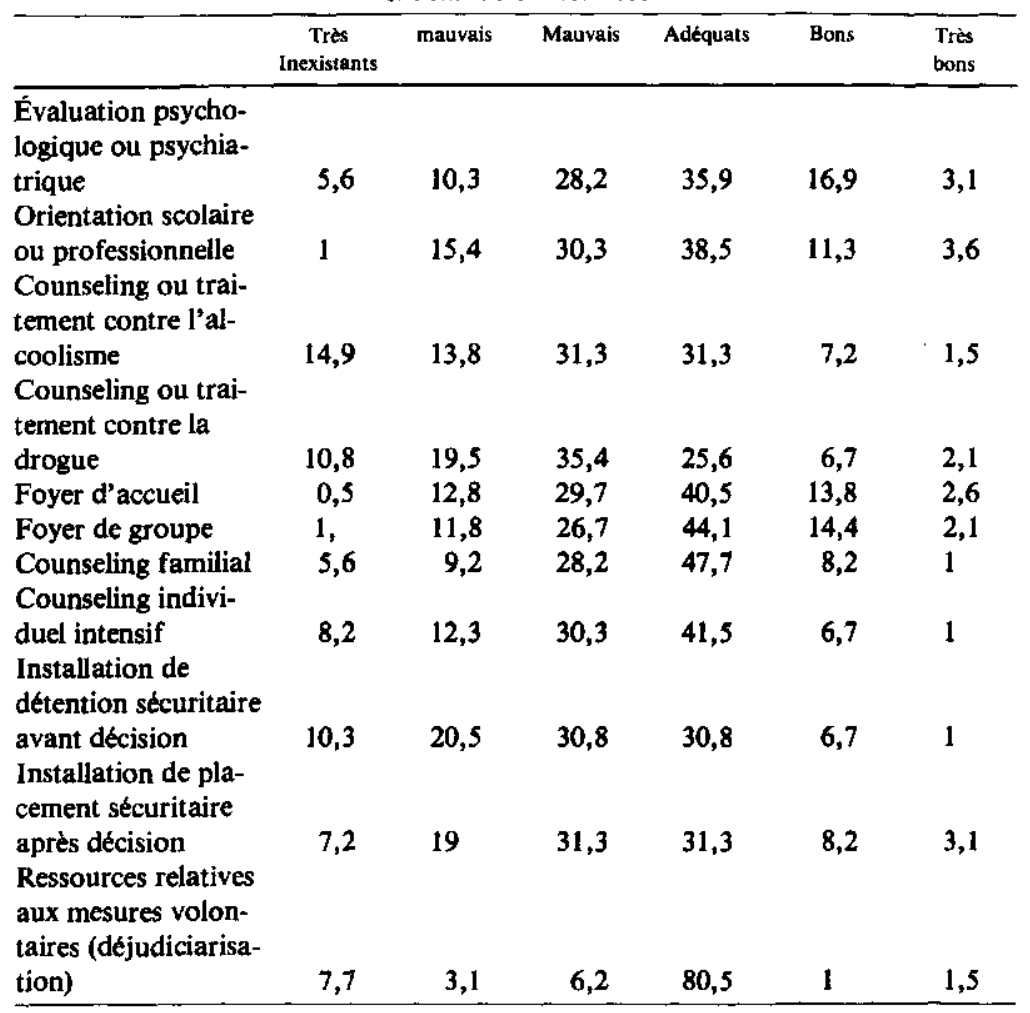

\section{BIBLIOGRAPHIE}

CHARBONNEAU, J.P. (1982), Rapport de la Commission parlementaire spéciale sur la protection de la jeunesse, Québec, Assemblée nationale du Québec, Direction générale des publications gouvernementales, ministère des Communications.

Code criminel, S.R.C. 1970, c. C-34.

FRÉCHETTE, M. et M. LEBLANC (1980), Pour une pratique de la criminologie: configurations de conduites délinquantes et portraits de délinquants, Montréal, Groupe de recherche sur l'inadaptation juvénile, cahier S, Inadaptation juvénile. 
GOTTFREDSON, M.R. and P.M. GOTTFREDSON (1982), Decision Making in Criminal Justice: Toward the Rational Exercise of Discusion, Cambridge, Ballenger Publishing Co.

KAPLAN, B. (1982), Points saillants de la Loi sur les jeunes contrevenants. Avantpropos.

LEBLANC, M. (1971), «La réaction sociale à la délinquance: une analyse stigmatique», Acto Criminologica, vol. IV, p. 113-192.

Loi sur les jeunes délinquants, S.R.C. 1970, c. J-3.

Loi sur les jeunes contrevenants, S.R.C. 1982, c. 61.

Loi sur la protection de la jeunesse, L.Q. 1977, c. 20.

MINISTĖRE DU SOLLICITEUR GÉNÉRAL (1982), La Loi sur les jeunes contrevenants: points saillants, Canada, Direction des Communications, Direction des programmes, Solliciteur général du Canada. 